\title{
The influence of socio demographic factors on severe weather concern, knowledge and preparedness among Malaysians
}

\begin{abstract}
The objective of this study was to examine the influence of socio-demographic factors on severe weather concern, knowledge and preparedness among urban and semi-urban residents of Malaysia. Results indicated that a broad-based education approach could be used to enhance community preparedness to severe weather risks as age, education level, gender and ethnicity did not influence all the variables of the study.
\end{abstract}

Keyword: Severe weather; Lightning; Lightning preparedness; Lightning education 